\title{
Tivozanib in advanced inoperable hepatocellular carcinoma: considerations for patients with liver cirrhosis
}

\author{
Hyunwoo Oh, Jeong-Hoon Lee
}

Department of Internal Medicine and Liver Research Institute, Seoul National University College of Medicine, Seoul, Republic of Korea Correspondence to: Jeong-Hoon Lee, MD, PhD. Department of Internal Medicine and Liver Research Institute, Seoul National University College of Medicine, 103 Daehak-ro, Jongno-gu, Seoul 03080, Republic of Korea. Email: pindra@empal.com; JHLeeMD@snu.ac.kr.

Comment on: Fountzilas C, Gupta M, Lee S, et al. A multicentre phase 1b/2 study of tivozanib in patients with advanced inoperable hepatocellular carcinoma. Br J Cancer 2020;122:963-70.

Submitted Apr 25, 2020. Accepted for publication May 26, 2020.

doi: $10.21037 /$ atm-20-3526

View this article at: http://dx.doi.org/10.21037/atm-20-3526

Liver cancer is the fourth leading cause of cancer-related death worldwide, with hepatocellular carcinoma (HCC) accounting for the majority of primary liver cancers (1). Approximately only $30-40 \%$ of patients with HCC are diagnosed at a very early or early stage according to the Barcelona Clinic Liver Cancer staging system, when potentially curative treatments (surgical resection, liver transplantation, or local ablation therapy) are employed as first-line treatment options (2). For patients with intermediate-stage HCC, locoregional therapies, including transarterial chemoembolization (TACE), can be applied. Patients who have advanced HCC with major vessel invasion, lymph node involvement, and/or extrahepatic metastasis or patients who experience progressive HCC refractory to TACE may be candidates for systemic therapy if underlying liver function and performance status are well preserved $(3,4)$. Unfortunately, more than half of patients with HCC are still diagnosed at an advanced stage globally (5). Thus, researchers and pharmaceutical companies have endeavored to understand HCC tumorigenesis, with tremendous developments and a change of paradigm in systemic therapies made following a number of trials. The use of sorafenib, as a systemic treatment initially led to significant improvement in overall survival (OS) among patients with advanced HCC (6). In the past few years, lenvatinib (7) has been approved as another firstline systemic treatment, while two programmed death-1 (PD-1) inhibitors [i.e., nivolumab (8), pembrolizumab (9), cabozantinib (10), and ramucirumab (11)] were approved as second-line treatment. However, the high incidence of underlying cirrhosis and the delayed diagnosis of HCC may interfere with the above treatments (3).

Meanwhile, tivozanib, an oral tyrosine kinase inhibitor (TKI), is a third-generation inhibitor of vascular endothelial growth factor receptor (VEGFR)-1, -2, and -3, showing high in vitro inhibitory activity against VEGFRs. Owing to favorable pharmacodynamic (PD) profiles in absorption, distribution, and elimination, tivozanib is expected to provide benefits in clinical practice (12). In a phase 3 trial, tivozanib therapy led to significantly superior progressionfree survival (PFS), objective response rate (ORR), and safety profiles in patients with metastatic renal cell carcinoma (RCC) compared to sorafenib (13). As the inhibition of VEGFRs is reportedly beneficial in HCC (14), tivozanib can be a potential candidate drug for HCC. However, considering a majority of HCC patients have underlying liver cirrhosis (3), it would be necessary to reestimate the optimal dose of tivozanib for such patients.

In a recent issue of British fournal of Cancer, Fountzilas and colleagues reported on the safety, dosing, pharmacokinetics (PK), PD, and anti-tumor activity of tivozanib in a total of 27 cirrhotic patients with advanced HCC whose liver function was well preserved as ChildTurcotte-Pugh (CTP) class A (15). In the present phase $1 \mathrm{~b} / 2$ study, considering results from a preceding phase 1 study of tivozanib on patients with various advanced, refractory solid tumors (16) and the presence of cirrhosis in included patients, the starting dose (level 1) was reduced from 1.5 to $1.0 \mathrm{mg}$. The maximum-tolerated dose and recommended phase 2 dose was determined to be $1.0 \mathrm{mg}$, 
once daily, for 21 days followed by 7 days off-treatment on a 28-day cycle through the phase $1 \mathrm{~b}$ part of this trial due to its dose-limiting toxicity. Median PFS and OS were 24 weeks and 9 months, respectively, and the ORR was $21 \%$ according to Response Evaluation Criteria in Solid Tumors 1.1 (15). This is the first study to report the response of tivozanib in patients with advanced HCC and cirrhosis.

On this issue, even though tivozanib failed to meet the pre-defined threshold (true progression rate of less than $\mathrm{P} 0=0.5$ ) and the investigators decided not to proceed to stage 2 of a phase 2 trial, this study is an important reference for second movers in estimating the safety and efficacy of tivozanib in patients with HCC. A sequel phase 1/2 trial of tivozanib in combination with durvalumab, a PD-1 ligand (PD-L1) inhibitor (NCT03970616), is recruiting patients since the ORR in patients treated with tivozanib was favorable. The synergistic effects of immune checkpoint inhibitors (ICIs) and angiogenesis inhibitors in combination with VEGFR inhibitors are drawing increasing attention after a very recent interim analysis of the IMbrave150 study showed a positive impact of atezolizumab (a PD-L1 inhibitor) and bevacizumab (a VEGFR inhibitor) on OS, PFS, and ORR over sorafenib. As a result, expected practice in firstline treatment for HCC may change (17). In a similar case, axitinib, which failed to improve OS over placebo in a phase 2 trial, was used to complete a phase 1 trial combination with avelumab (another PD-L1 inhibitor), showing a prolonged PFS and time to tumor progression (18).

Another attainment from the present study relates to safety. A recent network meta-analysis analyzing the safety of TKIs for RCC showed that tivozanib had a more favorable safety profile with a lower risk of grade 3 or 4 adverse events than other TKIs such as sorafenib, sunitinib, and cabozantinib (19). The authors observed the low incidence of severe liver toxicity, hand-foot-skin reactions, hypertension, and the absence of deaths due to toxicity (15). However, it is notable that the present study was conducted in patients with cirrhosis. In previous studies, including in patients with RCC, only patients with "sufficient" hepatic functional reservoir and those without chronic hepatitis $\mathrm{B}$ or $\mathrm{C}$ infection were included. All VEGFR-TKIs are mainly metabolized in the liver, especially by CYP3A4, although tivozanib is relatively less affected by CYP3A4 than other VEGFR-TKIs (12). CYP3A4, isoenzymes and their activity are reduced in patients with cirrhosis (20). In individual tivozanib exposure data suggested by the authors (supplement table 5), the maximum concentration for each patient was observed over a wide range which may have been due to different residual hepatic reservoir. Besides CYP3A4, several factors exist that may influence the PK/ PD profile, efficacy, and safety of TKIs in cirrhotic patients: portosystemic shunt, enterohepatic recirculation, biliary excretion, and drug reabsorption through gastrointestinal tract (21). In particular, in the case of patients with ascites (even in CTP class A6 cirrhosis), a portosystemic shunt with an increased hepatic venous pressure gradient resulted in the reduced first-pass metabolism of high-extraction ratio drugs and caused increased toxicity (22). In a previous study, patients with HCC underlying CTP class B cirrhosis under treatment with sorafenib showed significantly shorter survival and worse safety profiles compared to patients with CTP class A cirrhosis (23); moreover, a significant difference in OS existed even in the same CTP class A (5 vs. 6) (24).

In the present trial, the inclusion and exclusion criteria did not exclude unfavorable prognostic factors (i.e., $\geq 50 \%$ liver occupation, bile duct invasion, portal vein invasion, and lymph node involvement) that were excluded in the preceding phase 3 trials of TKIs in patients with HCC. Since the amount of immune suppressor cells (e.g., myeloid-derived suppressor cells, regulatory $\mathrm{T}$ cells, etc.) are positively associated with tumor volume, a larger tumor volume is known to be a predictor of a lower response rate and a worse prognosis (25). Thus, the clinical effect of tivozanib could be underestimated in the current study. As a sequel trial with tivozanib in combination with durvalumab (NCT03970616) had stricter selection criteria that excluded patients with tumor thrombus in the portal vein and inferior vena cava, the effect of tivozanib is expected to be better.

In summary, Fountzilas et al. suggested a dose of tivozanib for cirrhotic patients with HCC, which can be referred to by the next phase 2 study of tivozanib. Although the current study failed to proceed to stage 2 of a phase 2 trial, tivozanib showed a favorable response rate, PK/PD profiles, and safety profiles. We hopefully anticipate that tivozanib, in combination with durvalumab, will show a clinical benefit in a currently ongoing trial.

\section{Acknowledgments}

Funding: None.

\section{Footnote}

Provenance and Peer Review: This article was commissioned by the editorial office, Annals of Translational Medicine. The article did not undergo external peer review. 
Conflicts of Interest: Both authors have completed the ICMJE uniform disclosure form (available at http://dx.doi. org/10.21037/atm-20-3526). The authors have no conflicts of interests to declare.

Ethical Statement: The authors are accountable for all aspects of the work in ensuring that questions related to the accuracy or integrity of any part of the work are appropriately investigated and resolved.

Open Access Statement: This is an Open Access article distributed in accordance with the Creative Commons Attribution-NonCommercial-NoDerivs 4.0 International License (CC BY-NC-ND 4.0), which permits the noncommercial replication and distribution of the article with the strict proviso that no changes or edits are made and the original work is properly cited (including links to both the formal publication through the relevant DOI and the license). See: https://creativecommons.org/licenses/by-nc-nd/4.0/.

\section{References}

1. Yang JD, Hainaut P, Gores GJ, et al. A global view of hepatocellular carcinoma: trends, risk, prevention and management. Nat Rev Gastroenterol Hepatol 2019;16:589-604.

2. Llovet JM, Montal R, Villanueva A. Randomized trials and endpoints in advanced HCC: Role of PFS as a surrogate of survival. J Hepatol 2019;70:1262-77.

3. Forner A, Reig M, Bruix J. Hepatocellular carcinoma. Lancet 2018;391:1301-14.

4. Korean Liver Cancer Association; National Cancer Center. 2018 Korean Liver Cancer Association-National Cancer Center Korea Practice Guidelines for the Management of Hepatocellular Carcinoma. Gut Liver 2019;13:227-99.

5. Park JW, Chen M, Colombo M, et al. Global patterns of hepatocellular carcinoma management from diagnosis to death: the BRIDGE Study. Liver Int 2015;35:2155-66.

6. Llovet JM, Ricci S, Mazzaferro V, et al. Sorafenib in advanced hepatocellular carcinoma. N Engl J Med 2008;359:378-90.

7. Kudo M, Finn RS, Qin S, et al. Lenvatinib versus sorafenib in first-line treatment of patients with unresectable hepatocellular carcinoma: a randomised phase 3 noninferiority trial. Lancet 2018;391:1163-73.

8. El-Khoueiry AB, Sangro B, Yau T, et al. Nivolumab in patients with advanced hepatocellular carcinoma (CheckMate 040): an open-label, non-comparative, phase 1/2 dose escalation and expansion trial. Lancet. 2017;389:2492-502.

9. Zhu AX, Finn RS, Edeline J, et al. Pembrolizumab in patients with advanced hepatocellular carcinoma previously treated with sorafenib (KEYNOTE-224): a non-randomised, open-label phase 2 trial. Lancet Oncol 2018;19:940-52.

10. Abou-Alfa GK, Meyer T, Cheng AL, et al. Cabozantinib in patients with advanced and progressing hepatocellular carcinoma. N Engl J Med 2018;379:54-63.

11. Zhu AX, Kang YK, Yen CJ, et al. Ramucirumab after sorafenib in patients with advanced hepatocellular carcinoma and increased -fetoprotein concentrations (REACH-2): a randomised, double-blind, placebocontrolled, phase 3 trial. Lancet Oncol 2019;20:282-96.

12. Fogli S, Porta C, Del Re M, et al. Optimizing treatment of renal cell carcinoma with VEGFR-TKIs: a comparison of clinical pharmacology and drug-drug interactions of antiangiogenic drugs. Cancer Treat Rev 2020;84:101966.

13. Motzer RJ, Nosov D, Eisen T, et al. Tivozanib versus sorafenib as initial targeted therapy for patients with metastatic renal cell carcinoma: results from a phase III trial. J Clin Oncol 2013;31:3791-9.

14. Mossenta M, Busato D, Baboci L, et al. New insight into therapies targeting angiogenesis in hepatocellular carcinoma. Cancers (Basel) 2019;11:1086.

15. Fountzilas C, Gupta M, Lee S, et al. A multicentre phase $1 \mathrm{~b} / 2$ study of tivozanib in patients with advanced inoperable hepatocellular carcinoma. Br J Cancer 2020;122:963-70.

16. Eskens FA, de Jonge MJ, Bhargava P, et al. Biologic and clinical activity of tivozanib (AV-951, KRN-951), a selective inhibitor of VEGF receptor-1, -2 , and -3 tyrosine kinases, in a 4-week-on, 2-week-off schedule in patients with advanced solid tumors. Clin Cancer Res 2011;17:7156-63.

17. Lee M, Ryoo B, Hsu C, et al. Randomised efficacy and safety results for atezolizumab + bevacizumab in patients with previously untreated, unresectable hepatocellular carcinoma. Ann Oncol 2019;30:v851-934.

18. Kudo M, Motomura K, Wada Y, et al. First-line avelumab + axitinib in patients with advanced hepatocellular carcinoma: results from a phase $1 \mathrm{~b}$ trial (VEGF Liver 100). J Clin Oncol 2019;37:4072.

19. Manz KM, Fenchel K, Eilers A, et al. Efficacy and safety of approved first-line tyrosine kinase inhibitor treatments in metastatic renal cell carcinoma: a network meta-analysis. Adv Ther 2020;37:730-44. 
20. Villeneuve JP, Pichette V. Cytochrome P450 and liver diseases. Curr Drug Metab 2004;5:273-82.

21. Weersink RA, Burger DM, Hayward KL, et al. Safe use of medication in patients with cirrhosis: pharmacokinetic and pharmacodynamic considerations. Expert Opin Drug Metab Toxicol 2020;16:45-57.

22. Verbeeck RK. Pharmacokinetics and dosage adjustment in patients with hepatic dysfunction. Eur J Clin Pharmacol 2008;64:1147-61.

23. Marrero JA, Kudo M, Venook AP, et al. Observational registry of sorafenib use in clinical practice across

Cite this article as: $\mathrm{Oh} \mathrm{H}$, Lee $\mathrm{JH}$. Tivozanib in advanced inoperable hepatocellular carcinoma: considerations for patients with liver cirrhosis. Ann Transl Med 2020;8(22):1530. doi: 10.21037/atm-20-3526
Child-Pugh subgroups: the GIDEON study. J Hepatol 2016;65:1140-7.

24. Kim HY, Park JW, Joo J, et al. Worse outcome of sorafenib therapy associated with ascites and Child-Pugh score in advanced hepatocellular carcinoma. J Gastroenterol Hepatol 2013;28:1756-61.

25. Lee JH, Lee JH, Lim YS, et al. Adjuvant immunotherapy with autologous cytokine-induced killer cells for hepatocellular carcinoma. Gastroenterology 2015;148:1383-91.e6. 